\title{
Review of: "Mechanisms for destabilisation of RNA viruses at air-water and liquid-liquid interfaces"
}

Jie Zhuang ${ }^{1}$

1 University of Tennessee, Knoxville

Potential competing interests: The author(s) declared that no potential competing interests exist.

This is a very important topic about virus inactivation at various interfaces. The mechanisms of inactivation at the air-water interfaces indeed remains unclear due to technical difficulty in microscopic experimental observation. My main concern about the article is on the correction of the hypothesis --- interfacial electrostatic forces can cause inactivation of negatively charged viruses. Our macroscopic experiments demonstrated that electrostatic forces play a much smaller role in deactivating negatively charged viruses, compared to chemical ions (especially cations such as $\mathrm{Ca} 2+$ and $\mathrm{HCO} 3-$ ). The effect of air-water interfaces depends on solution chemical conditions and/or physiological responses of viruses to the chemical ions. Thus, this published theoretical simulation needs experiential support. In addition, viral morphology must be considered in the simulation process. For instance, the "hairs" on the capsid might carry positive charges or have a different zeta potential, and these hairs also influence capillary forces that pin viruses at the air-water interfaces. 